Ann. Zootech., I978, 27 (4), 495-517.

\title{
Observations préliminaires sur quelques effets du lactose chez le porc : Complexes myoélectriques de l'intestin grêle, motricité caecale et diarrhée
}

\author{
J. P. LAPLACE \\ avec la collaboration technique de C. GERMAIN \\ Laboratoive de Physiologie de la Nutrition, \\ Centre national de Recherches zootechniques, I.N.R.A., \\ 78350 Jouy-en-Josas, France
}

\section{Résumé}

I,es effets de l'ingestion d'un régime semi-synthétique comportant $82 \mathrm{p}$. I oo de lactose pur à l'égard de l'organisation des activités électriques de l'intestin grêle, ont été étudiés chez 5 porcs de race Large White, pesant en moyenne $57 \mathrm{~kg}$ et équipés d'électrodes chroniques intrapariétales souples. Un sixième porc a subi une fistulation réentrante du jéjunum distal pour vérifier et évaluer l'effet de ce régime sur les masses de digesta en transit.

L'organisation des activités rapides au niveau duodénal reste normale. Mais la migration en direction aborale des phases consécutives des complexes myoélectriques est largement perturbée. Au niveau jéjuno-iléal, des activités régulières naissent et s'effacent de façon relativement anarchique. Globalement on enregistre une raréfaction des activités régulières, pouvant atteindre sur l'iléon une absence totale pendant des périodes de $5 \mathrm{~h}$ au moins à $2 \mathrm{r}$ h au plus. Cet effet est d'autant plus prolongé que la quantité de lactose ingéré est plus importante. Parallèlement l'intensité des activités rapides irrégulières est considérablement amoindrie. On observe cependant dans le jéjunum le transit rapide d'un volume de digesta $z$ fois plus important qu'avec un régine standard, et 3 à 4 fois plus qu'avec un aliment à base d'amidon de maîs.

Ces perturbations ne peuvent être interprêtées de façon simple ni par un effet insulinique, ni par le seul effet de réplétion intestinale tel qu'il se manifeste dans d'autres conditions d'alimentation.

\section{Introduction}

L'activité lactasique du tube digestif, autorise chez de nombreux jeunes mammifères une parfaite utilisation du lait maternel. I a lactase présente en effet une activité élevée à la naissance chez le Rat (KoLdovsky et Chytil, I965), 1a Souris (YeH et Moog, I974), ou le Porc (Bailey, Kitts et Woods, I956; Walker, I959; Hartman et al., I96I; Dahiquist, I96I; Manners et St'Tenens, I972). Dans 
toutes ces espèces, l'activité lactasique diminue au cours de 1'allaitement (KoIDovsky, I966). Cette réduction s'effectue chez le porcelet jentre la naissance et 1'âge de 8 semaines (MANNERS et STEVENS, I972). L'activité lactasique n'évolue plus guère ensuite jusqu'à l'âge adulte. Elle est alors trop faible pour que l'animal supporte et digère une ration très riche en lactose sans intolérances digestives et limitation de ses performances (EKSTROM, Grummer, BenEvenga, 1976).

Cette situation n'est pas susceptible d'amendement puisque l'incorporation de lactosérum à l'aliment, pour des périodes prolongées, n'entraîne aucune modification de l'activité lactasique de la muqueuse de l'intestin grêle du Porc (EiksTROM, BENEVENGA, Grummer, I975). La lactase endogène s'avère donc être une enzyme non adaptative au substrat, même face à des régimes contenant $60 \mathrm{p}$. Ioo de lactosérum (Aumaitre, FÉvrier, Dewulf, I977). La malabsorption irréductible du lactose ingéré en grandes quantités est ainsi la principale responsable des diverses manifestations d'intolérance, en dépit d'une forte élévation de l'activité lactasique totale au niveau des contenus du gros intestin et d'une très importante hydrolyse du lactose par la flore gastro-intestinale du Porc (Aumaitre, FÉvrier, DEWULF, I977). Les résultats apportés par KERN et STRUTHERS (I966), LAUNIALA (I968) et Debongnie, Newcomer et Phillips (1977) dans le cas de l'Homme déficient en lactase, suggèrent une accélération du transit dans 1'intestin grêle lors d'ingestion de lactose.

Ce travail a pour objectif : a) une première analyse des effets du lactose à l'égard des complexes myoélectriques de l'intestin grêle du Porc; $b$ ) une évaluation préliminaire du rôle du lactose en tant que tel, et de celui de l'appel osmotique qu'il induit, dans les phénomènes observés; $c$ ) l'obtention d'informations relatives à la motricité caecale et à l'apparition de la diarrhée dans la perspective d'une exploration ultérieure des relations entre phénomènes moteurs et diarrhée.

\section{Matériel et méthodes}

\section{Animaux et préparation chirurgicale}

Six porcs de race Large White, d'un poids initial de $57,4 \pm \mathrm{I}, 8 \mathrm{~kg}$, codés J-K-L-M-N et $\mathrm{O}$, sont utilisés pour cette étude. Chez 5 d'entre eux, des électrodes d'acier inoxydable sont implantées, sous anesthésie générale, dans les couches musculaires de l'intestin grêle et éventuellement du cæcum, par groupes de 3 en chaque site, selon la technique précédemment décrite (LAPLACE, I978). Le porc O est équipé d'une fistule réentrante jéjunale en un site dont la distance par rapport au pylore représente $66 \mathrm{p}$. Ioo de la longueur de l'intestin grêle. Les canules sont réalisées et mises en place selon les techniques décrites par LAPLACE et TOMASSONE (I970). Le poids vif des porcs lors de l'opération, leur gain moyen quotidien pendant la période expérimentale, et la localisation des sites d'électrodes sur l'intestin grêle sont détaillés dans le tableau I.

\section{Aliments et modes de distribution}

L'aliment de base, utilisé pour l'obtention des enregistrements de référence, est un aliment standard de croissance (tableau 2) auquel les porcs sont accoutumés depuis le sevrage. Il est systématiquement distribué à raison de 2 repas par jour $(9 \mathrm{~h}$ et $\mathrm{I} 6 \mathrm{~h}$ 30) sous forme de soupe liquide (farine à $88 \mathrm{p}$. Ioo de matière sèche 


\section{TABLEAU I}

Poids vif lors de l'opération, gain moyen quotidien (G.M.Q.) pendant la période expérimentale, et localisation des sites d'électrodes sur l'intestin grêle (en p. too de la longueur totale de celui-ci) pour les 5 porcs utilisés au cours de l'étude électromyographique.

Pigs used for electromyographical studies: live weight at the time of surgery, mean daily weight gain (M.D.G.) during experimentation period, and localization of the electrode groups on the small bowel (as p. Ioo of total smali bowel length).

\begin{tabular}{|c|c|c|c|}
\hline $\begin{array}{c}\text { Pore } \\
\text { Pig }\end{array}$ & $\begin{array}{l}\text { Poids vif } \\
\text { Live weight } \\
\quad(\mathrm{kg})\end{array}$ & $\begin{array}{l}\text { G.M.Q. } \\
\text { M.D.G. } \\
(\mathrm{g} / \mathrm{j}) \\
(g / d)\end{array}$ & $\begin{array}{c}\text { Localisation des sites d'électrodes } \\
\text { Localization of the electrodes } \\
\text { (p. IOo) }\end{array}$ \\
\hline $\begin{array}{l}\mathrm{J} \\
\mathrm{K} \\
\mathrm{I} \\
\mathrm{M} \\
\mathrm{N}\end{array}$ & $\begin{array}{l}57,0 \\
65,0 \\
57,0 \\
58,5 \\
52,0\end{array}$ & $\begin{array}{l}529 \\
645 \\
703 \\
368 \\
530\end{array}$ & $\begin{array}{l}99, \mathrm{I}-99,3-99,5-99,8 \\
97,7-98,3-98,9-99,5-99,9 \\
97,5-98,2-98,9-99,6-99,9 \\
12,4-25,2-38,0-49,5-61,1 \\
72,6-85,4-98,3- \\
8,3-20,5-30,5-42,6-52,8 \\
63,2-75,7-90,1-9\end{array}$ \\
\hline
\end{tabular}

TABLEAU 2

Composition centésimale des aliments utilisés

Percentual composition of the feeds used

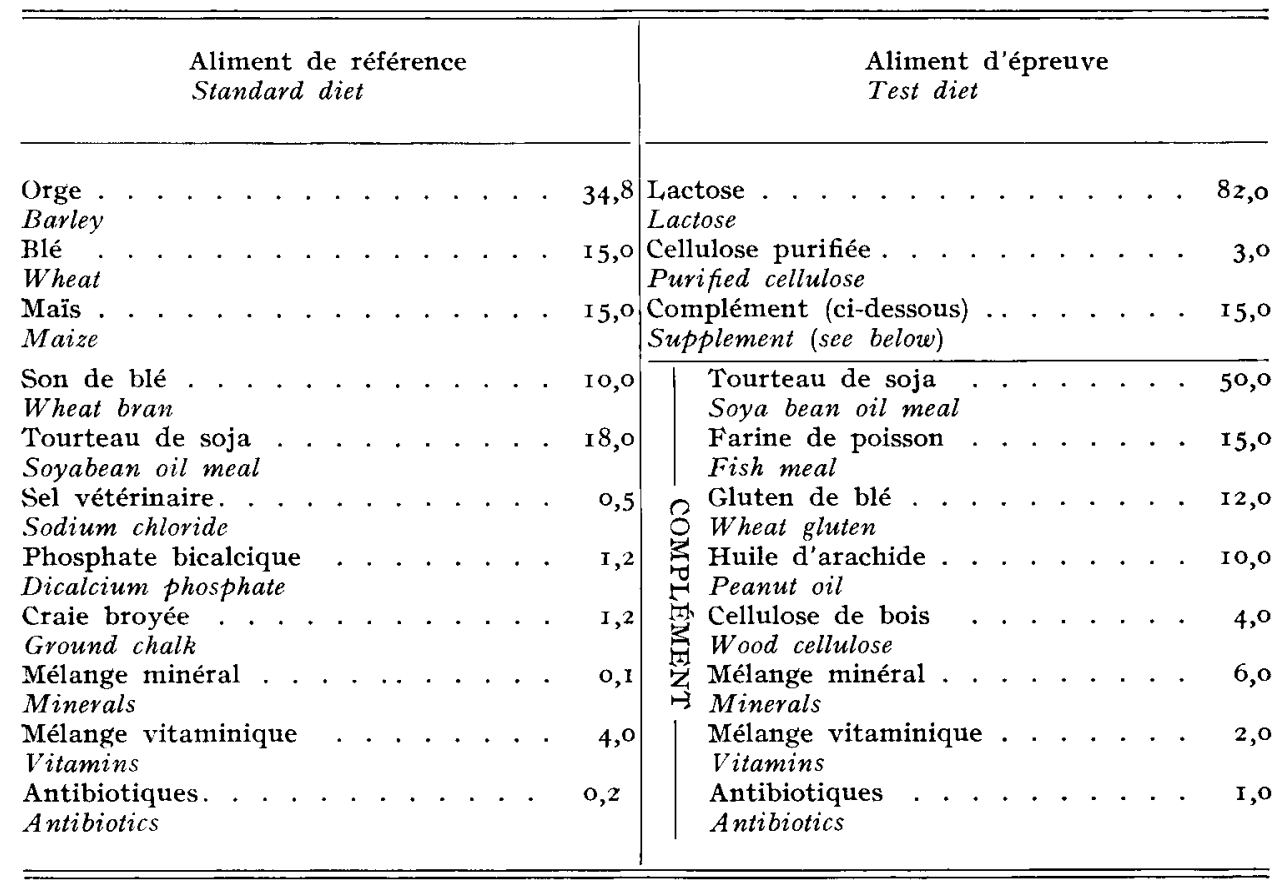


diluée par 2 fois son poids d'eau). Une ration de I ooo à I $200 \mathrm{~g}$ de farine fraîche présentée sous cette forme est ingérée en 6 à $8 \mathrm{mn}$.

Les épreuves au lactose consistent en la substitution, sans habituation préalable, des 2 repas d'aliment standard d'un même jour par 2 repas d'un aliment semi-synthétique contenant $82 \mathrm{p}$. Ioo de lactose (tabl. z). Celui-ci, à l'état de farine, est présenté après dilution par un même poids d'eau. Son acceptabilité moindre conduit à des temps d'ingestion légèrement allongés $\left(\begin{array}{llll}8 & \text { à } 2 & m n & \text { pour }\end{array}\right.$ I $200 \mathrm{~g}$ de farine dans les meilleurs cas), ou très augmentés chez d'autres porcs (I5 à $20 \mathrm{mn}$ pour $800 \mathrm{~g}$ de farine). Cet aliment semi-synthétique riche en lactose est identique, pour la fraction assurant les apports protéique, cellulosique, minéral et vitaminique, à l'aliment semi-synthétique riche en amidon de maïs antérieurement utilisé (IAPLACE, I 978). Les effets de ce dernier justifient qu'il ne soit pas retenu ici comme aliment de référence et que nos électromyogrammes témoins soient réalisés après ingestion de l'aliment standard usuel pour porcs en croissance.

Chez le porc $O$, la mesure directe, au cours des $6 \mathrm{~h}$ post-prandiales, des volumes de digesta transitant au niveau de la fistule jéjunale, a été réalisée pour les 3 régimes standard, semi-synthétique-lactose et semi-synthétique-amidon de maïs.

Les porcs disposent dans tous les cas en permanence d'eau à volonté.

\section{Conditions expérimentales}

Toutes les conditions générales de l'expérience sont rigoureusement identiques à celles décrites antérieurement (LAPLACE, I978). Après 8 jours de récupération post-opératoire et d'accoutumance au nouvel environnement, les enregistrements sont effectués durant des périodes continues de $48 \mathrm{~h}$ en régime de référence ou de $72 \mathrm{~h}$ selon la séquence suivante $: 24 \mathrm{~h}$ en régime de référence, puis $24 \mathrm{~h}$ avec 2 repas d'épreuve au lactose, et à nouveau $24 \mathrm{~h}$ en régime de référence. L'intervalle entre 2 nycthémères d'épreuve au lactose a été de 6 jours au moins et 22 jours au plus, chaque animal ayant subi au plus 2 ou 3 épreuves.

\section{Enregistrements}

Les activités myoélectriques sont enregistrées sur un polygraphe 8 voies (Dynograph RM, BECKMAN) en utilisant des constantes de temps très brèves $(0,03 \mathrm{sec}$.$) de façon à réduire les variations lentes de potentiel et à privilégier$ les salves de potentiels d'action. Les activités intestinales ainsi recueillies sont enregistrées et/ou traitées selon les 3 modalités dont le détail a été antérieurement exposé (LAPLACE, I978) : a) électromyogramme global direct, b) électromyogrammes direct et intégré en parallèle (intégration linéaire selon LATOUR, I973),c) électromyogramme intégré avec conversion en mode digital selon FAYART, DABURON et LAPLACE, I978).

\section{Résultats}

\section{I. - Les complexes myoélectriques lors d'ingestion du régime standard}

Rappelons simplement ici qu'un complexe myoélectrique comporte successivement une phase I dite de quiescence, une phase II dite d'activité de pointes irrégulière et une phase III dite d'activité de pointes régulière. Une brève phase IV, 
intermédiaire entre cette dernière activité et la période de quiescence, peut éventuellement être distinguée. La définition de chacune de ces phases a été apportée de façon très précise par CoDE et MARLETT (I975) chez le Chien. Cette terminologie et les définitions correspondantes sont applicables au Porc pour lequel une description détaillée des caractéristiques spécifiques des complexes myoélectriques a été récemment publiée (LAPLACE, I978).

Les données de référence recueillies dans le présent travail s’inscrivent bien dans le tableau précédemment apporté (LAPLACE, I978) : au niveau de l'intestin grêle proximal on enregistre pour les pores $M$ et $N 20$ à 24 complexes en $24 \mathrm{~h}$, d'une durée moyenne de 60 à $70 \mathrm{mn}$, lors d'ingestion du régime standard; les durées moyennes de chacune des 3 phases constitutives sont comprises, selon les nycthémères et le porc concernés, entre I I et 2 I mn (quiescence), 42 à $45 \mathrm{mn}$ (activité irrégulière) et $4 \mathrm{mn}$ (activité régulière). Ces mêmes valeurs au niveau de l'intestin grêle distal sont (moyenne des valeurs moyennes respectivement obtenues pour chacun des enregistrements témoins de chaque porc) : I I4,2 mn (séquence $\mathrm{I}+\mathrm{II}+\mathrm{III}$ ), 33,6 $\mathrm{mn}$ (quiescence), 77, I $\mathrm{mn}$ (activité irrégulière), 3,7 mn (activité régulière) pour I2 complexes en $24 \mathrm{~h}$.

La caractéristique fondamentale des complexes myoélectriques naissant périodiquement sur le duodénum est de migrer lentement en direction aborale, au moins pour la majorité d'entre eux. L'estimation, chez les porcs $M$ et $N$, de la vitesse moyenne de franchissement de la totalité de l'intestin grêle (longueur totale I $560 \mathrm{~cm}$ chez M, I I $88 \mathrm{~cm}$ chez N), sur la base de la migration des activités régu. lières enregistrées au cours des $24 \mathrm{~h}$, fournit des valeurs moyennes de référence de $9,6 \mathrm{~cm} / \mathrm{mn}(\mathrm{M})$ et $8,3 \mathrm{~cm} / \mathrm{mn}(\mathrm{N})$. Ceci correspond à un temps total théorique de franchissement de l'intestin grêle par une activité régulière de $162 \mathrm{mn}$ (M) ou I $44 \mathrm{mn}(\mathrm{N})$, pour des temps réels mesurés de I60,5 $\pm 3,3 \mathrm{mn}$ ( $n=$ Io, Porc $\mathrm{M}$ ) et de $137,8 \pm 7,6 \mathrm{mn}(n=5$, Porc $\mathrm{N})$.

\section{2. - Modifications par le lactose des électromyogrammes de l'intestin grêle}

L'influence du lactose à l'égard des activités électriques rapides (potentiels d'action) de l'intestin grêle s'avère relativement variable dans le degré des modifications induites. Deux dominantes sont constamment observées : a) une hypomotilité intestinale i.e. une forte réduction de l'intensité des activités rapides irrégulières; b) une perturbation de la migration proximodistale des complexes i.e. en particulier l'effacement de nombreuses activités régulières. L,es différences de degré dans ces effets selon les porcs ru tudent pratiquement impossible l'expression systématique de valeurs moyennes, en dépit de l'homogénéité constante dans la nature des changements qui apparaissent.

\section{a) Nature et siège des modifications}

Au niveau de 1'intestin grêle proximal, le nombre total de complexes enregistrés en $24 \mathrm{~h}$ lors d'ingestion de lactose (fig. I - AI) n'est jamais modifié de façon significative par rapport aux valeurs de référence en régime standard (fig. I-BI). De même, les valeurs moyennes exprimant pour les porcs $M$ et $N$ la durée des complexes et de chacune des phases qui les constituent, lors d'ingestion de lactose, sont normales : $64,7 \pm 3,2 \mathrm{mn}$ (séquence I + II + III), I6,I $\pm 2,2$ mn (quiescence), $43,3 \pm 4,8 \mathrm{mn}$ (activité irrégulière), $4, \mathrm{I} \pm 0, \mathrm{I} \mathrm{mn}$ (activité régulière). 


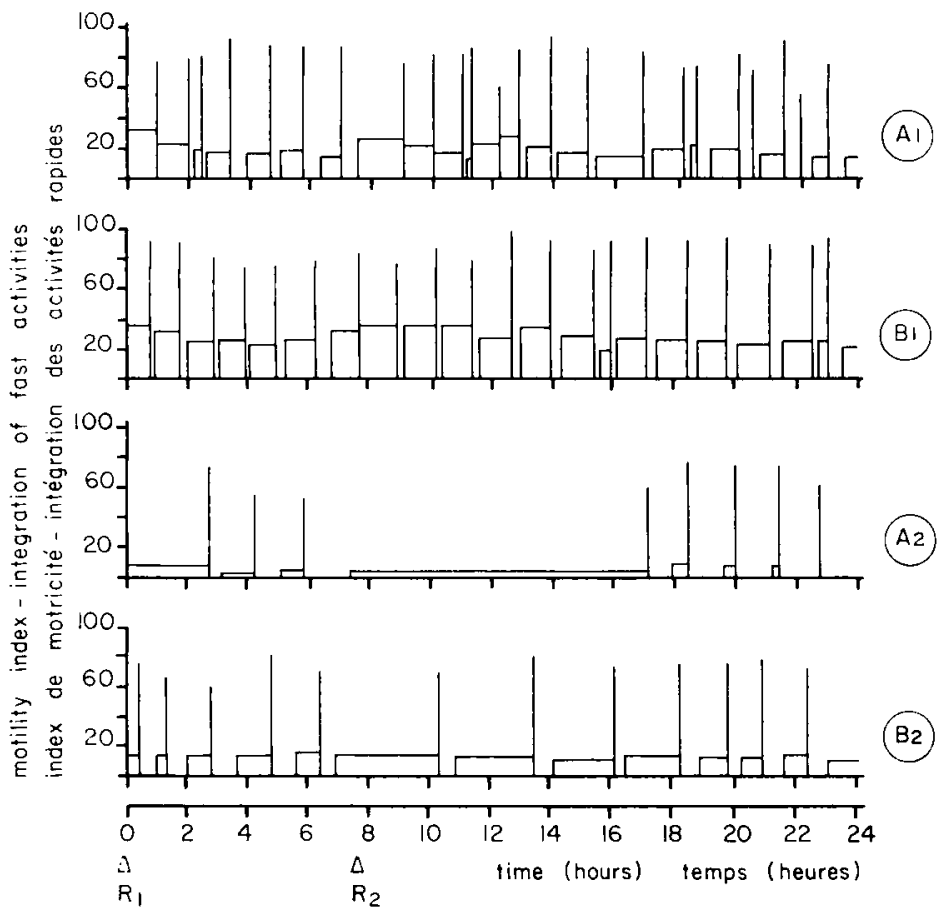

FIG. I. - Représentation graphique de la succession des complexes myoélectriques au cours d'un cycle de 24 h au niveau du duodénum $\left(A_{1}\right.$ et $B_{1}$ à $I 93 \mathrm{~cm}$ en aval du pylore) et de l'iléon $\left(A_{2}\right.$ et $B_{2} \grave{a}_{I} 533 \mathrm{~cm}$ du pylore) chez le Porc selon la nature de l'aliment ingéré. La hauteur des rectangles (activités irrégulières) et celle des barres (activités régulières) est proportionnelle à la hauteur moyenne des pics d'intégration exprimant l'activité intestinale pendant la durée de la phase considérée.

$A_{1}$ et $A_{2}=$ Nycthémère "Lactose " avec une ingestion représentant la consommation effective de $400 \mathrm{~g}$ de lactose pur à $9 \mathrm{~h}\left(\mathrm{R}_{1}\right)$ et de $800 \mathrm{~g}$ de lactose pur à $\mathrm{r} 6 \mathrm{~h} 30\left(\mathrm{R}_{\mathrm{z}}\right)$. Noter l'absence d'activités régulières sur l'iléon pendant $5 \mathrm{~h}$ et la faible amplitude moyenne de activités irrégulières.

$B_{1}$ et $B_{2}=$ Nycthémère suivant avec ingestion de $\mathrm{I}$ ooo $\mathrm{g}$ d'aliment standard à chacun des 2 repas.

FIG. I. - Graph of the migrating myoelectrical complexes during a 24 h cycle on the duodenum $\left(A_{1}\right.$ and $B_{1}-I 93 \mathrm{~cm}$ distal to the pylorus $)$ and on the ileum $\left(A_{2}\right.$ and $B_{2}-I 533 \mathrm{~cm}$ distal to the pylorus) of the Pig according to the diet. The height of the rectangles (irregular spiking activities) and that of the bars (regular spiking activities) is proportional to the mean height of the integration peaks recorded during the phase concerned.

$A_{1}$ and $A_{2}=$ Intake of $400 \mathrm{~g}$ of pure lactose (in the form of the semi synthetic diet) at 900 a.m $\left(\mathrm{RI}_{\mathrm{I}}\right.$ and of $800 \mathrm{~g}$ of pure lactose at $4.30 \mathrm{p} . \mathrm{m}$. $\left(\mathrm{R}_{2}\right)$. Note the absence of any regular spiking activity on the ileum during $5 \mathrm{~h}$ and the low mean amplitude of the irregular spiking activities.

$\mathrm{B}_{1}$ and $\mathrm{B}_{2}=$ Next day, intake of $\mathrm{x}$ ooo $\mathrm{g}$ standard diet at each of the 2 meals.

L'examen des électromyogrammes (fig. 2) permet par ailleurs de constater que l'organisation post-prandiale habituelle des activités rapides duodénales fait défaut lors d'ingestion de lactose. L'activité de pointes irrégulière en région proximale reste, même durant la première heure qui suit l'ingestion du repás, extrêmement clairsemée (fig. 2A).

Au niveau de l'intestin grêle distal, et par rapport à ce qui est enregistré en régime standard (fig. I-B2), l'ingestion de lactose conduit à observer (fig. I-A2) 


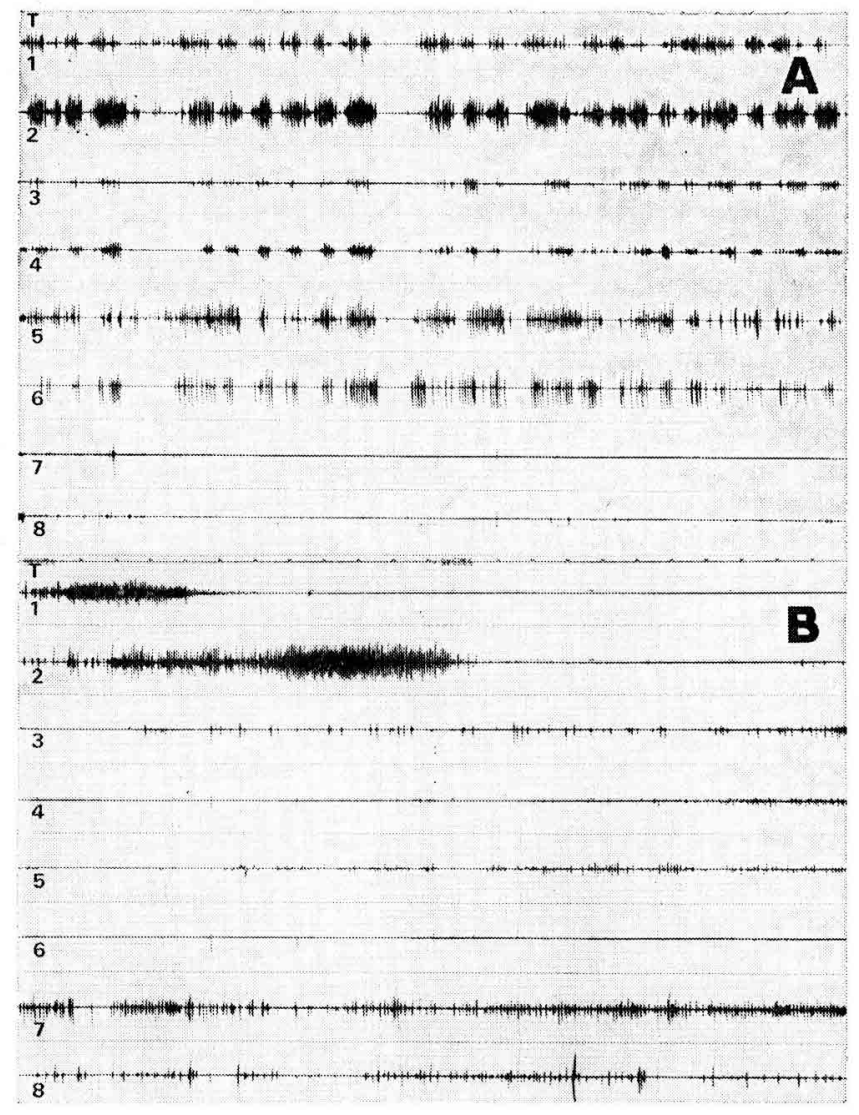

FIG. 2. - Electromyogramme global vecueilli, chez un porc consommant un végime semi-synthétique $\grave{a}$ base de lactose, en 8 sites échelonnés au long de l'intestin grêle, respectivement à une distance du pylove de $193 \mathrm{~cm}(\mathrm{I}), 393 \mathrm{~cm}(2), 593 \mathrm{~cm}(3), 773 \mathrm{~cm}(4), 953 \mathrm{~cm}$ (5), I I33 cm (6), I $333 \mathrm{~cm}$ $(7)$, et $r 533 \mathrm{~cm}(8)$, pour une longueur totale de l'intestin grêle de $1560 \mathrm{~cm}$. A la partie supérieure de chaque enregistrement, la base de temps ( $\mathrm{T}$ ) indique la minute.

En $A=50$ mn après le premier repas à $9 \mathrm{~h}$ représentant la consommation de $700 \mathrm{~g}$ de lactose pur. On remarque en (I) que l'activité irrégulière n'est pas surchargée par le pattern post prandial habituel.

$\mathrm{Fn} B=9 \mathrm{~h} 20 \mathrm{mn}$ après le second repas à $16 \mathrm{~h} 30 \mathrm{mn}$ représentant également l'ingestion de $7^{\circ} \mathrm{o} \mathrm{g}$ de lactose pur. Des activités régulières (visibles en I et 2 ) existent et migrent en région proximale, mais sont totalement absentes en région distale. Les activités irrégulières sont très faibles aux niveaux (3) à (8).

FIG. 2. - Electromyographic activities recorded in 8 intestinal sites, located $193 \mathrm{~cm}(\mathrm{I}), 393 \mathrm{~cm}(2)$, $593 \mathrm{~cm}(3), 773 \mathrm{~cm}(4), 953 \mathrm{~cm}(5), I I 33 \mathrm{~cm}(6), I 333 \mathrm{~cm}(7), I 533 \mathrm{~cm}(8)$ distal to the pylorus, respectively. The total small bowel length was I $560 \mathrm{~cm}$ in this $P i g$, which was fed a semisynthetic diet including lactose. The time marker ( $\mathrm{T})$ on the top of each panel shows the minute.

Panel $\mathrm{A}=50 \mathrm{~min}$. after the first meal at $9.00 \mathrm{a} . \mathrm{m}$. allowing a $700 \mathrm{~g}$ pure lactose intake. It may be noted ( $\mathrm{I}$ ) that irregular spiking activity is not overlapped by the usual post prandial pattern.

Panel $B=9 \mathrm{~h}$ zo min. after the second meal at $4.3^{\circ} \mathrm{p} . \mathrm{m}$. also allowing a $700 \mathrm{~g}$ pure lactose intake. Regular spiking activities can be seen in ( $(\mathbf{r})$ and (2), migrating over the proximal small bowel. They fail to appear in distal area. Irregular spiking activities are very low at levels (3) to $(8)$. 
d'une part une forte réduction du nombre de séquences complètes, la disparition de plusieurs activités régulières et du silence consécutif conduisant à un allongement apparent de la phase d'activité irrégulière, et d'autre part une forte diminution de l'intensité des activités de pointes irrégulières sur la base de l'intégration des bouffées de potentiels d'action. L'intestin grêle distal est donc le plus affecté à la suite de l'ingestion de lactose. Il présente une importante hypomotilité.

Ce phénomène d'hypomotilité s'avère, à l'examen d'électromyogrammes dérivés en des sites échelonnés tout au long de l'intestin grêle, concerner l'ensemble du jéjuno-iléon. A la suite du premier repas à base de lactose, on assiste à une raréfaction des bouffées de potentiels d'action, dont l'importance (nombre et amplitude des potentiels) décroît parallèlement. On assiste alors à la migration éventuelle (selon le site étudié - voir $\$ c$ ci-après) d'activités régulières à la suite de longues périodes au cours desquelles il est presque impossible de délimiter valablement une phase de quiescence et une phase d'activité irrégulière tant les tracés sont plats (fig. $2 \mathrm{~B}$ ). Cet état d'hypomotilité est toujours persistant. Des activités irrégulières normales ne sont retrouvées qu'à la suite du repas d'aliment standard du lendemain matin.

\section{b) Électromyogramme iléal et quantité de lactose ingéré}

L'hypomotilité iléale, sur la base des activités de pointes irrégulières, est rendue très évidente par l'intégration des activités rapides (sites 3 et 4 - fig. $3 \mathrm{~A}$ ). Mais indépendamment de cet aspect commun à tous les pores, 1'analyse de la chronologie du déroulement des complexes après ingestion de lactose amène à observer deux ordres de grandeur dans la durée de la période pendant laquelle toute activité de pointes régulière fait défaut sur l'iléon (fig. $2 \mathrm{~B}$ et $3 \mathrm{~B}$ ).

Dans les cas d'ingestion modérée de lactose (I $300 \mathrm{~g}$ au plus pour les $24 \mathrm{~h}$ ), l'aversion de l'animal pour l'aliment ayant raison de son appétit, 7 à 8 activités régulières seulement sont constatées sur l'iléon en $24 \mathrm{~h}$, le plus long intervalle entre elles atteignant exceptionnellement ro h. Ainsi, dans le cas du porc M (illustré par la fig. I-A2), pour une ingestion de $400+800 \mathrm{~g}$ de lactose pur (sous forme de l'aliment semi-synthétique complet), la durée moyenne pour les $24 \mathrm{~h}$ d'une phase d'activité irrégulière atteint $132,9+77,7 \mathrm{mn}$. Cette valeur anormalement élevée et le coefficient de variation associé sont la conséquence de l'absence de toute activité régulière, et de la quiescence consécutive, durant environ Io $\mathrm{h}$. Chez le Porc $\mathrm{L}$, après ingestion de $400+900 \mathrm{~g}$ de lactose pur, la durée moyenne pour les $24 \mathrm{~h}$ d'une phase d'activité irrégulière est de $\mathrm{r}_{56,6} \pm 34$, I $\mathrm{mn}$, l'absence de toute activité régulière n'excédant guère $5 \mathrm{~h}$.

Lors de consommation importante d'aliment semi-synthétique, représentant au total I 500 à $2000 \mathrm{~g}$ de lactose pur ingéré en 2 repas, 1'absence de toute activité régulière au niveau iléal est beaucoup plus durable. Ainsi toute activité régulière est absente durant i $6 \mathrm{~h}$ ( $2000 \mathrm{~g}$ de lactose pur en 2 repas) et $\mathrm{I} 6 \mathrm{~h} 30$ (I $500 \mathrm{~g}$ de lactose pur en 2 repas) chez le porc $\mathrm{K}$; cette même disparition est constatée durant plus de $2 \mathrm{I} \mathrm{h}$ ( $\mathrm{I} 600 \mathrm{~g}$ de lactose pur en 2 repas) ou 2 fois Io h consécutives ( $600 \mathrm{~g}$ de lactose pur en 2 repas) chez le porc J. Dans ces cas d'absence très prolongée des activités régulières sur l'iléon après ingestion de grandes quantités de lactose, leur réapparition peut revêtir deux formes. Soit les activités régulières se manifestent d'emblée avec leur périodicité usuelle en ce site distal, et leur nombre total pour le nycthémère d'épreuve au lactose est particulièrement faible (3 phases III). Soit on 


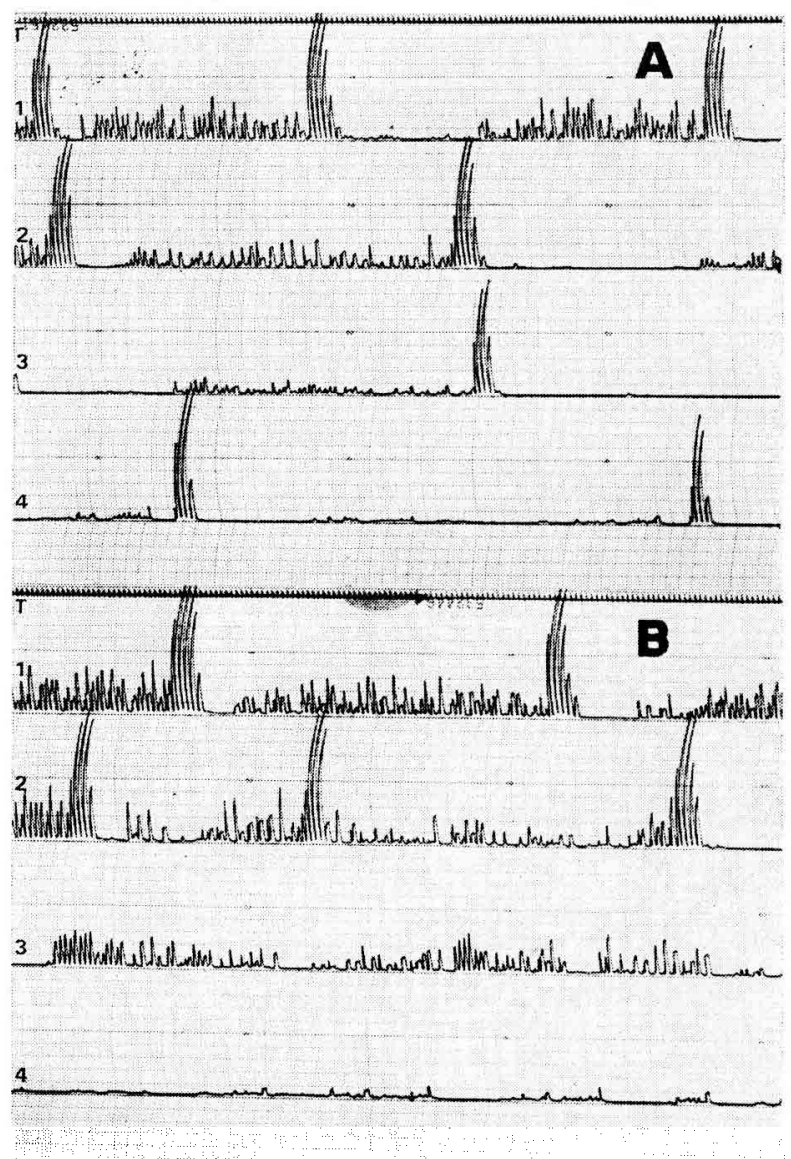

FIG. 3. - Intégration linéaire par unité de temps de I $m n$ des potentiels d'action recueillis en 4 sites intestinaux, respectivement situés à $193 \mathrm{~cm}(\tau), 773 \mathrm{~cm}(2), I 333 \mathrm{~cm}(3), I 533 \mathrm{~cm}$ (4) du pylore, chez le même porc que dans la figure 2. A la partie supérieure de chaque enregistrement, la base de temps (T) indique la minute. L'aspect normal de tels envegistrements intégrés a été décrit dans un précédent article (LAPLACE, 1978).

En $\mathrm{A}=2 \mathrm{~h} 50 \mathrm{mn}$ après le premier repas à $9 \mathrm{~h}$ représentant la consommation de $400 \mathrm{~g}$ de lactose pur. La réduction importante voire disparition des activités irrégulières est observable en (2), (3) et (4).

En $B=7 \mathrm{~h}$ après le second repas à $\mathrm{i} 6 \mathrm{~h} 30$ représentant la consommation de $800 \mathrm{~g}$ de lactose pur. On note sur les sites distaux (3 et 4 ) l'absence de toute phase d'activité régulière.

FIG. 3. - Linear integration per minute of spike potentials recorded from 4 intestinal sites, located

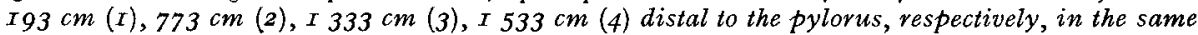
Pig as in figure 2 . The time marker (T) on the top of each panel shows the minute. The normal features of such integrated records were described in a previous paper (LAPLACE, I978).

Panel $\mathrm{A}=2 \mathrm{~h} 50 \mathrm{~min}$. after the first meal at $9.00 \mathrm{a} . \mathrm{m}$. allowing a $400 \mathrm{~g}$ pure lactose intake. The large decrease and even disappearance of irregular spiking activities can be seen in (2), (3) and (4).

Panel $\mathrm{B}=7 \mathrm{~h}$ after the second meal at $4.30 \mathrm{p} . \mathrm{m}$. allowing a $800 \mathrm{~g}$ pure lactose intake. It may noted that any regular spiking activity fails to appear in distal bowel area ( 3 and 4 ). 
assiste à une multiplication exceptionnelle des activités régulières conduisant à un nombre total pour les $24 \mathrm{~h}$ de l'ordre de 7 à 8 phases III. C'est ainsi que l'on voit chez le porc $K$, après $I 6 \mathrm{~h}$ consécutives d'absence, se succéder 7 activités régulières à des intervalles anormalement brefs pour un site iléal, à savoir $20,40,15,45,50$ et $70 \mathrm{mn}$.

\section{c) Migration des complexes myoélectriques au long de l'intestin grêle}

L'absence plus ou moins prolongée des phases d'activité régulière au niveau de l'iléon suggère que la migration des complexes myoélectriques dans le sens proximo-distal est perturbée lors d'ingestion de lactose. En pratique, les phases I de quiescence et II d'activité irrégulière migrent tout comme les phases III;

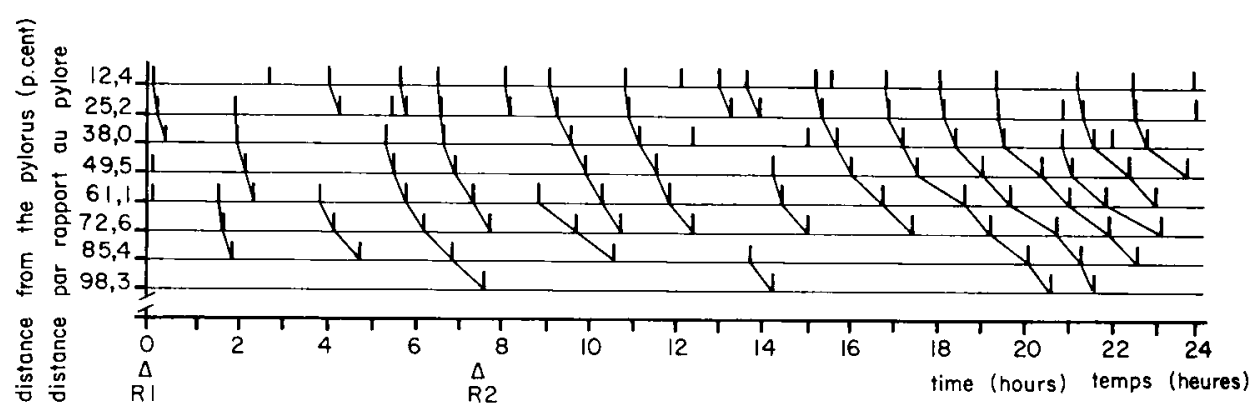

FIG. 4. - Migration des phases d'activité réguliève (barrettes verticales) le long de l'intestin grêle durant un cycle de 24 h chez un porc ayant ingévé 2 vepas $\left(\mathrm{R}_{1} \grave{a} 9\right.$ h et $\mathrm{R}_{2} \dot{a} I 6$ h 30$)$ représentant chacun un apport de $700 \mathrm{~g}$ de lactose pur. Ce graphique peut être comparé aux graphiques homologues en régime standard et amidon de maïs présentés dans un précédent article (LAPLACF, I978) et réalisés à partir d'enregistrements recueillis chez le même porc.

Chaque ligne cortespond à un site d'enregisttement dont la distance par rapport au pylore, indiquée en ordontiée, est exprimée en p. roo de la longueur totale de l'intestin grêle.

FIG. 4. - Migration of regular spiking activity phases (vertical bars) along the small bowel during a 24 h cycle in a Pig which ingested 2 meals $\left(\mathrm{R}_{1}\right.$ at 9.00 a.m. and $\mathrm{R}_{2}$ at 16.30 p.m.) each of them allowing a $700 \mathrm{~g}$ pure lactose intake. This graph can be compared to the homologous graphs for standard and maize starch diets published in a previous paper (LAPLACF, 1978), and done from records obtained in the same pig. Each line of the graph corresponds to a recording site, identified by its distance from the pylorus (ordinate), as percent of total small bowel length

mais l'hypomotilité jéjuno-iléale rend trop arbitraire la délimitation des phases I et II pour qu'une évaluation précise de leur migration soit obtenue. La migration des activités régulières est donc seule analysée ici.

La représentation graphique (fig. 4) des activités régulières enregistrées en $24 \mathrm{~h}$ au niveau de sites échelonnés au long de l'intestin grêle illustre la perturbation, retrouvée dans tous les cas, de leur migration. Les activités régulières qui franchissent la totalité de l'intestin grêle sont rares au cours des $I 8 \mathrm{~h}$ à $20 \mathrm{~h}$ après le premier repas de lactose. De plus on enregistre la naissance apparemment aléatoire de telles phases en n'importe quel point du jéjunum, leur migration restant limitée à 2 ou 3 sites consécutifs d'enregistrement.

Malgré ces modifications, la vitesse moyenne de franchissement de la totalité de l'intestin grêle peut être estimée chez les porcs $M$ et $N$ porteurs d'électrodes échelonnées tout au long du viscère. Les activités régulières en registrées au cours 
des périodes de $24 \mathrm{~h}$ d'épreuve au lactose migrent à des vitesses sensiblement plus élevées ( $\mathrm{I}, \mathrm{I} \mathrm{cm} / \mathrm{mn}$, porc $\mathrm{M}-\mathrm{II}, 4 \mathrm{~cm} / \mathrm{mn}$, porc $\mathrm{N}$ ) que celles précédemment indiquées pour les enregistrements de référence en régime standard. Cependant, cette accélération globale de la migration n'est pas constante. Elle est variable selon le site intestinal considéré et l'éloignement plus ou moins grand du repas.

Au niveau du quart proximal de l'intestin grêle, la migration est en fait, pour l'ensemble des complexes du nycthémère, systématiquement ralentie avec le lactose (en moyenne $26,6 \mathrm{~cm} / \mathrm{mn}$ au lieu de 35,4 en régime standard). Pour tout le territoire jéjunal (sites 25 à $75 \mathrm{p}$. IOO) une accélération globale est constatée en moyenne sur les complexes des $24 \mathrm{~h}(8,9$ contre $7,7 \mathrm{~cm} / \mathrm{mn})$. Mais cette accélération est en réalité le fait des migrations en période diurne (I0,3 contre $7,2 \mathrm{~cm} / \mathrm{mn}$ ), une tendance au ralentissement apparaissant à l'inverse en période nocturne $(7,9$ contre $8,4 \mathrm{~cm} / \mathrm{mn})$. Au niveau iléal en fin, l'accélération en période diurne n'est retrouvée que de façon inconstante; par contre, en période nocturne, c'est-à-dire d'éloignement croissant de l'ingestion de lactose, le ralentissement de la migration des complexes est important. Cet effet est particulièrement évident chez les porcs J et $\mathrm{K}$ pour lesquels les activités régulières ne reparaissent que $\mathrm{I} 6$ à $2 \mathrm{I} \mathrm{h}$ après le premier repas de lactose. Dans ce cas on enregistre sur l'iléon des vitesses telles que en moyenne $2,4 \mathrm{~cm} / \mathrm{mn}$ (porc J) ou $2,8 \mathrm{~cm} / \mathrm{mn}$ (porc $\mathrm{K}$ ) contre respectivement 5,0 et $5,2 \mathrm{~cm} / \mathrm{mn}$ en régime standard.

Les différences des vitesses de migration au cours des périodes diurne et nocturne et l'absence durant plusieurs heures de toute migration proximodistale complète, interdisent l'obtention d'un temps total moyen de franchissement de l'intestin grêle par une activité régulière. En pratique, il n'a été possible qu'une setule fois de mesurer ce temps total en période diurne, chez le Porc N. La valeur obtenue - $95 \mathrm{mn}$ - est très nettement inférieure au temps total moyen de référence chez le même porc (I37,8 mn), ce qui confirme l'accélération générale de la migration, telle qu'évaluée pour des migrations partielles. A l'inverse en période nocturne, la réapparition tardive de migrations complètes permet de vérifier leur ralentissement au travers d'un temps moyen de $173,0+15,7 \mathrm{mn}(n=3)$ contre I60,5 $\pm 3,3 \mathrm{mn}$ en régime standard $(n=1$ o) chez le Porc $\mathrm{M}$, et de $164,0 \pm 5,2 \mathrm{mn}$ $(n=3)$ contre $137,8 \pm 7,6(n=5)$ chez le Porc $\mathrm{N}$.

\section{3. - Evaluation du transit des digesta dans l'intestin grêle}

L,es collectes des contenus digestifs arrivant au dernier tiers de l'intestin grêle (fig. 5) montrent que l'ingestion de lactose conduit à des volumes de digesta présents dans la lumière intestinale beaucoup plus importants qu'avec le régime standard et plus encore qu'avec un régime amidon de maïs. Cependant, pour éva-luer correctement ces différences, il convient de tenir compte du volume réel de la ration diluée et de l'eau bue au cours de la période post-prandiale de collecte. En effet, les aliments semi-synthétiques sont dilués par un poids d'eau équivalent, alors que l'aliment standard l'est par un poids d'eau double de celui de farine. Par ailleurs l'abreuvement spontané est toujours plus important à la suite des repas de lactose. Le tableau 3 fournit, pour les 3 repas d'épreuve illustrés dans la figure 5 , les diverses valeurs caractéristiques de l'ingéré, et l'écart du volume collecté en $6 \mathrm{~h}$ au volume ingéré total en p. Ioo de ce dernier. Schématiquement, et à ingéré équivalent, on peut retenir que le volume de digesta collectés en $6 \mathrm{~h}$ au niveau de la canule jéjunale est deux fois plus important après un repas de lactose qu'après un 


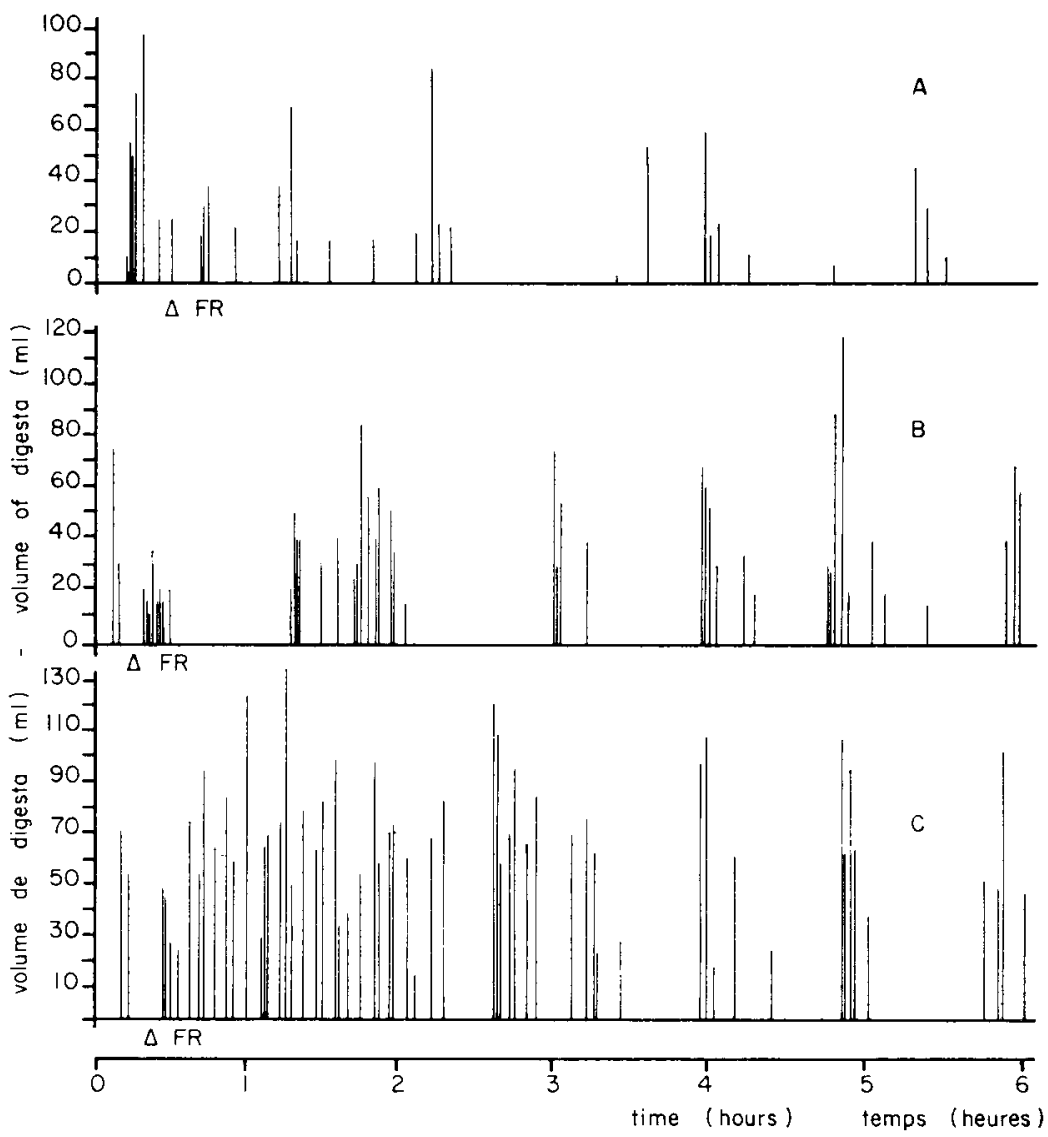

FIG. 5. -- Représentation schématique, chronologique et quantitative, de l'écoulement du contenu jéjunal chez le Porc, au niveau d'une fistule réentrante placée en un site dont la distance au pylore $(865 \mathrm{~cm})$ représente 66 p. 100 de la longueur de l'intestin. Collecte totale des digesta assurée manuellement avec restitution immédiate à l'animal de chaque émission après lecture de son volume.

En $A=$ après ingestion d'aliment semi-synthétique à base d'amidon de maïs.

En $B=$ après ingestion d'aliment standard.

En $\mathrm{C}=$ après ingestion d'aliment semi-synthétique à base de lactose.

Les volumes d'aliment dilué et d'eau de boisson ingérés, et les volumes de digesta collectés pour ces 3 épreuves sont indiqués dans le tableau 3 .

$\mathrm{FR}=$ fin du repas d'épreuve.

FIG. 5. - Graphs showing the rate of jejunal content outflow (chronology and volume) in the Pig, from a veentrant cannula located $865 \mathrm{~cm}$ distal to the pylorus i.e. 66 p. 100 of the total small bowel length. Manual collection of digesta with immediate return to the Pig of each gush after reading of its volume.

Graph $A=$ after intake of a maize starch semi synthetic diet.

Graph $B=$ after intake of a standard diet.

Graph $C=$ after intake of a lactose semi synthetic diet.

The volumes of diluted feed and of water ingested, and the volumes of digesta collected during each of these 3 tests are indicated in table 3 .

$\mathrm{FR}=$ End of the test meal. 
TABLEAU 3

Volumes caractéristiques de l'ingéré total pour chacune des épreuves, illustrées dans la figuve 5 , de collecte pendant $6 \mathrm{~h}$ des digesta émis au niveau d'une canule jéjunale véentrante chez le Porc. Volumes totaux correspondants de digesta collectés et différence par rapport à l'ingéré (variation en p. Ioo par rapport au volume total ingéré)

Characteristic volumes of the total intake during each digesta collection tests, described by figure 5 , done during 6 h from a jejunal reentrant cannula in the Pig. Total corresponding volumes of digesta collected and difference as related to intake (variation as $p$. Ioo of total ingested volume)

\begin{tabular}{|c|c|c|c|c|c|c|c|}
\hline Régime & $\begin{array}{l}\text { Quantité } \\
\text { d'aliment }\end{array}$ & $\begin{array}{c}\text { Eau } \\
\text { de }\end{array}$ & $\begin{array}{l}\text { Volume } \\
\text { de la }\end{array}$ & $\begin{array}{l}\text { Eau } \\
\text { bue }\end{array}$ & $\begin{array}{l}\text { Volume } \\
\text { total }\end{array}$ & \multicolumn{2}{|c|}{$\begin{array}{l}\text { Volume de digesta } \\
\text { Volume of digesta }\end{array}$} \\
\hline Diet & $\begin{array}{c}\text { (g) } \\
\text { Quantity } \\
\text { of flour } \\
(\mathrm{g})\end{array}$ & $\begin{array}{l}\text { du repas } \\
\text { (ml) } \\
\text { Water } \\
\text { dilution } \\
\text { of the } \\
\text { flour } \\
(\mathrm{ml})\end{array}$ & $\begin{array}{l}\text { diluée } \\
\text { (ml) } \\
\text { lolume } \\
\text { of the } \\
\text { diluted } \\
\text { meal } \\
(\mathrm{ml})\end{array}$ & $\begin{array}{c}\text { (ml) } \\
\\
\text { Water } \\
\text { intake in } \\
6 \mathrm{~h} \\
(\mathrm{ml})\end{array}$ & $\begin{array}{l}\text { (ml) } \\
\text { Total } \\
\text { ingested } \\
\text { volume } \\
(\mathrm{ml})\end{array}$ & $\begin{array}{c}\text { Collectés } \\
\text { en } 6 h(\mathrm{ml}) \\
\text { collected } \\
\text { in } 6 h \\
(\mathrm{ml})\end{array}$ & $\begin{array}{c}\text { Variation } \\
p . \text { Ioo } \\
\text { du volume } \\
\text { ingévé } \\
p . \text { Ioo } \\
\text { of the } \\
\text { volume } \\
\text { ingested }\end{array}$ \\
\hline $\begin{array}{l}\text { Amidon de mais } \\
\text { Maize starch }\end{array}$ & 780 & 780 & I $24^{\circ}$ & I IOO & $234^{\circ}$ & 956 & $-59,2$ \\
\hline $\begin{array}{l}\text { Standard . . . . } \\
\text { Standard }\end{array}$ & 600 & I 200 & I $63^{\circ}$ & I 000 & 2630 & I 899 & $-z_{7,8}$ \\
\hline $\begin{array}{l}\text { Lactose } \\
\text { Lactose }\end{array}$ & 980 & 980 & I $64^{\circ}$ & I 600 & 3240 & 4397 & $+35,7$ \\
\hline
\end{tabular}

repas d'aliment standard. Par rapport au volume collecté après ingestion d'amidon de maïs, un volume $I, 5$ à 2 fois plus élevé est obtenu après un repas d'aliment standard, et 3 à 4 fois plus élevé après un repas de lactose.

\section{4. - Activités électromyographiques du cacum et diarrhée}

Chez 3 porcs (J, K et $L_{\text {) }}$ les électromyogrammes du cæecum ont été simultanément enregistrés, à titre indicatif des modifications de l'activité du gros intestin après ingestion de lactose. Il convient cependant de ne pas oublier que l'ensemble du territoire colique représente 2,5 à $3 \mathrm{~m}$ d'intestin en aval du cæcum, et donc que celui-ci ne peut être tenu a priori pour représentatif de l'ensemble.

Le repas du matin est toujours associé à une forte hypermotilité cæacale pendant environ I h (II à I3 bouffées de potentiels par période de $6 \mathrm{mn}$ ). Au delà, le nombre moyen de bouffées de potentiels en $6 \mathrm{mn}$ est de $6,7 \mathrm{chez}$ le porc ayant consommé l'aliment standard. Par contre, après ingestion de lactose en grande quantité (800 à I ooo $\mathrm{g}$ pour un repas), l'activité cæcale est fortement réduite entre la $2^{\mathrm{e}}$ et la 8 e heure post-prandiale (en moyenne 2,6 bouffées de potentiels par $6 \mathrm{mn}$ ). Cette réduction est moins accusée pour des consommations moindres de lactose 

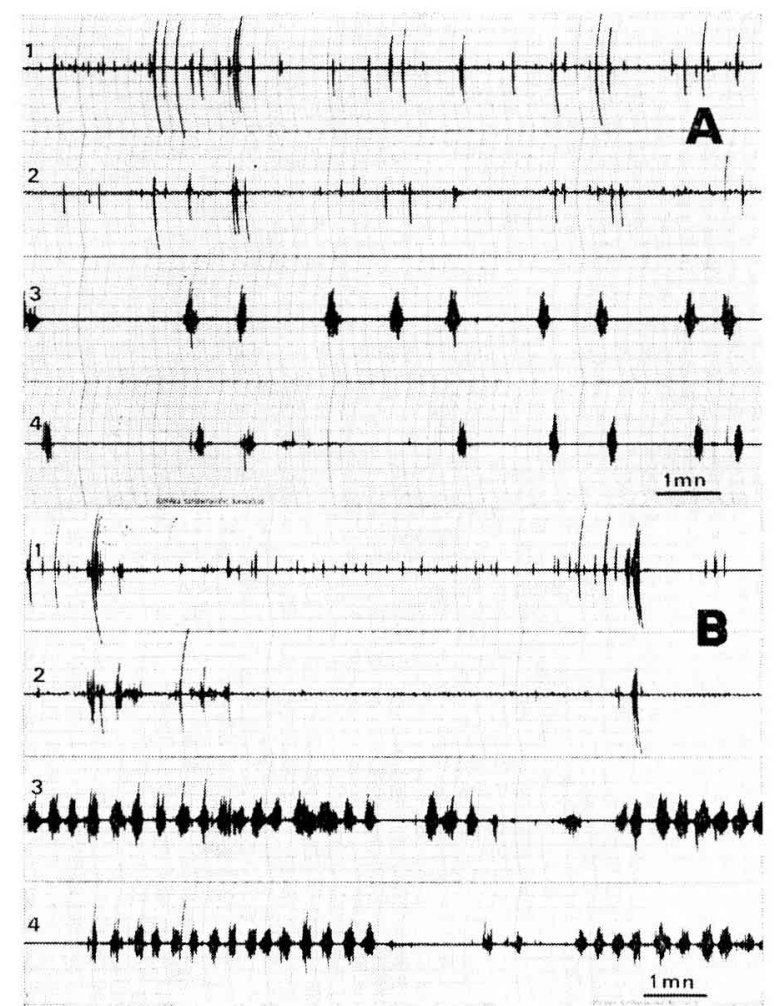

Fig. 6. - Activités électriques iléo-cocales après ingestion de lactose chez le Porc. Sur chaque enregistrement on trouve les électromyogrammes de l'iléon à $10 \mathrm{~cm}(1)$ et à $5 \mathrm{~cm}(2)$ de la jonction iléocacale, et ceux du cxcum à $5 \mathrm{~cm}$ (3) et à $10 \mathrm{~cm}$ (1) de cette même jonction.

En $\mathrm{A} \ldots 2 \mathrm{~h} 30 \mathrm{mn}$ après un repas à $9 \mathrm{~h}$ représentant l'ingestion de $500 \mathrm{~g}$ de lactose pur. Les activités irrégulières iléales sont déjà très réduites. La période d'hypomotilité cæcale débute à ce stade.

En $B=I 3 h 3^{\circ} \mathrm{mn}$ après ce même repas, soit $6 \mathrm{~h}$ après le second repas à I 6 h 30 qui représente l'ingestion de I ooo $g$ de lactose pur. Les activités irrégulières iléales sont toujours très faibles. I'hypermotilité cæcale a succédé à l'hypomotilité des 8 premières heures. La diarrhée n'est pas encore apparue à ce stade.

FIG. 6. - Electrical activities of the ilcum and cocum after lactose intake in the Pig. Each panel shows the electromyograms of the ileum at $10 \mathrm{~cm}(\mathrm{I})$ and $5 \mathrm{~cm}(2)$ above the ileo-cocaljunction, and those of the cacum at $5 \mathrm{~cm}$ (3) and $10 \mathrm{~cm}$ (4) beyond this junction.

Panel $\mathrm{A}=2 \mathrm{~h} 30 \mathrm{~min}$. after a meal at 9.00 a.m. allowing a $500 \mathrm{~g}$ pure lactose intake. Trregular spiking activities were already reduced on the ileum. The caecal hypomotility is now appearing.

Panel $\mathrm{B}=\mathrm{I} 3 \mathrm{~h} 30 \mathrm{~min}$. after this same meal, i.e. $6 \mathrm{~h}$ after a second meal at $4 \cdot 3 \circ$ p.m. allowing a $\mathrm{I}$ ooo $\mathrm{g}$ pure lactose intake. Ileal irregular spiking activities are always low. Cæcal spike bursts are numerous, probably indicating an hypermotility. Diarrhoea did not appear at this time.

(400 à $600 \mathrm{~g}$ pour un repas). Au-delà de la $8^{\mathrm{e}}$ heure postprandiale, on assiste à l'inverse à 1'apparition d'épisodes d'hypermotilité cæcale (jusqu'à 20 bouffées par $6 \mathrm{mn}$ ) dont la multiplication conduit à une hyperactivité quasi continue. Les premières manifestations de 1'hypomotilité cæcale surviennent parallèlement à la réduction de l'intensité des activités irrégulières iléales (fig. 6A). L’hyperactivité 
cæcale ultérieure coexiste avec l'absence d'activité régulière et la persistance d'activités irrégulières très faibles sur l'iléon (fig. 6B).

La diarrhée consécutive à l'ingestion de lactose, systématiquement observée dans les conditions expérimentales et pour les animaux ici utilisés, apparaît généralement 15 à $20 \mathrm{~h}$ après le premier repas d'épreuve au lactose. Par contre, lors d'ingestion modérée de lactose (cas du porc $\mathrm{L}$, avec suppression des activités régulières iléales durant $5 \mathrm{~h}$ seulement) les premières fèces spumeuses ne sont émises que 23 à $24 \mathrm{~h}$ après le premier repas d'épreuve et sont suivies après $30 \mathrm{mn}$ d'un bref épisode diarrhéique.

\section{Discussion}

L'insuffisance de lactase endogène peut être considérée comme la cause primitive des troubles diarrhéiques observés chez le Porc, comme pour d'autres espèces, après l'ingestion d'une grande quantité de lactose. Ce phénomène a été initialement attribué par WEIJERS et al. (I96I) à l'effet des produits acides résultant de la fermentation du lactose dans le côlon. L'acide lactique et autres acides organiques à courte chaîne d'origine fermentaire, responsables de l'acidification des contenus coliques, seraient à l'origine de l'augmentation du péristaltisme et de l'excrétion d'eau vers la lumière intestinale (Rousseau et SLAden, r97r; Christopher et BAYLESS, I97I). Ils sont en effet relativement mal absorbés par le côlon de certains monogastriques (PARsons et PATERson, I965; HELLER et KERN, I968), et s'accumulent donc dans les contenus coliques. Dans le cas particulier du Porc, RERAT (I975) observe que les quantités d'acide lactique absorbées après un repas de lactose ne sont pas négligeables. Filles restent néanmoins deux fois plus faibles qu'après ingestion d'amidon de maïs. Mais il semble, au moins dans ce dernier cas, que l'acide lactique apparu dans le sang de la veine porte puisse résulter de la métabolisation de certains glucides dans la paroi digestive. Les informations actuellement disponibles ne permettent donc pas d'évaluer la production d'acide lactique dans le côlon du Porc après ingestion de lactose, ni l'absorption de cet acide lactique issu d'une digestion microbienne.

Cette pathogénie strictement colique a été élargie par la démonstration du rôle important de l'intestin grêle, dans lequel le lactose non hydrolysé est par luimême responsable d'une sécrétion nette d'eau et d'une accélération du transit (KERN et STRUThers, I 966; LAUNiala, I968). L'importance du volume de liquide présent dans le jéjunum, dont l'abreuvement accru ne peut à lui seul rendre compte, témoigne, chez nos pores, de la réalité de cet effet osmotique du lactose dans l'intestin grêle, tel qu'explicité par Christopher et Bayless (I97I). En définitive, ces perturbations au niveau de l'intestin grêle apparaissent comme fort importantes et les principales responsables des phénomènes douloureux de crampes abdominales, connus chez l'Homme (Christopher et BAyless, I97I).

\section{I. - Aspects méthodologiques}

La variabilité relativement importante entre porcs du nombre de complexes myoélectriques entegistrés en $24 \mathrm{~h}$ (LAPLACE, I978) rend nécessaire 1'utilisation de chaque porc comme son propre témoin. L'homogénéité de nature et de sens des effets observés, lorsqu'elle est vérifiée, autorise la présentation de données 
moyennes traduisant globalement l'influence étudiée. Cependant une difficulté d'un autre type est rencontrée dans ce travail, en raison des très grandes différences dans l'ampleur de certains des effets du lactose. La répartition des réponses en deux classes de durée de disparition des activités régulières au niveau iléal peut être attribuée en première analyse à l'in fluence de la quantité de lactose effectivement ingérée. Mais il est impossible en toute rigueur de dissocier cet effet quantité d'un effet porc puisque certains animaux ne consomment systématiquement que des quantités limitées de lactose, tandis que d'autres porcs en ingèrent lors de chaque épreuve une grande quantité. Or l'existence d'une susceptibilité variable de la musculature intestinale à l'égard du lactose (par quelque mécanisme que ce soit) ne peut être exclue a priori.

Un autre aspect méthodologique qui limite la portée de ces premiers résultats doit être souligné. L'aliment semi-synthétique apportant le lactose a été distribué sous la forme de repas d'épreuve sans accoutumance préalable, à la différence de ce qui a pu être fait pour le régime amidon de maïs (LAPLACE, I978) utilisable comme aliment quotidien pour des périodes prolongées. Mais il paraît exclu d'utiliser ce type d'aliment semi-synthétique à haute teneur en lactose pur durant plusieurs jours consécutifs, sans risquer de perturber l'état clinique des animaux. De même ce régime ne se prêtant pas à la granulation ou à une distribution sous forme sèche, ne peut être distribué ad libitum. Ceci revient à dire que les résultats obtenus ne peuvent être étendus sans réserve à d'autres aliments, contenant du lactose, mais utilisables de façon continue et auxquels le tube digestif pourrait éventuellement s'accoutumer. Il sera donc intéressant de réexaminer la motricité de l'intestin grêle chez des porcs ingérant quotidiennement un aliment à base de lactosérum sec par exemple.

Cette autre situation expérimentale recourant à un aliment sec présenté sous forme granulée, au même titre qu'un aliment standard, devrait également permettre de faciliter les comparaisons en supprimant les différences de taux de dilution de la farine avant présentation, et de fluidité de la soupe ingérée.

Un dernier écueil de la méthodologie utilisée, résulte de la composition respective des régimes confrontés. La fraction lactose de l'aliment semi-synthétique n'est pas la seule différence par rapport à l'aliment standard, qui reste pourtant la véritable référence normale. Ce problème justifie que 1'interprétation des effets du lactose soit réalisée autant par rapport à ceux obtenus avec l'aliment standard que par rapport à ceux observés pour l'aliment amidon de maïs (LAPLACE, I978) comportant les mêmes fractions complémentaires que l'aliment lactose.

\section{2. - Hypomotilité de l'intestin grêle}

La réduction globale des activités rapides (potentiels d'action) de l'intestin grêle après ingestion de lactose est une constante des effets observés. Deux aspects peuvent être distingués : a) l'absence en région proximale de l'organisation postprandiale habituelle; b) la diminution considérable des activités irrégulières et l'absence en certains sites, principalement distaux, des activités régulières.

\section{a) Absence d'hyperactivité post-prandiale}

Chez le Chien (Ruckebusch et Fioramontr, r975) comme chez le Mouton (BUENO, WEEKES et RUCKEBUSCH, I977) l'insuline a été considérée comme la principale responsable de l'apparition d'une période post-prandiale prolongée 
d'intense activité irrégulière, masquant l'organisation normale des complexes en séquences consécutives. Le rôle de l'insuline est apparu également plausible dans le cas du Porc consommant un régime standard ou un régime semi-synthétique à base d'amidon de maïs (LAPLACE, I978), qui apportent l'un comme l'autre de grandes quantités de sucres simples dans la circulation portale. Le nerf pneumogastrique paraît jouer également un certain rôle dans l'hyperactivité post-prandiale, au moins chez le Chien (RUCKEBUSCH et BUENo, I977). Celle-ci est en effet retardée chez l'animal vagotomisé, et sa durée se trouve réduite. Chez le Chien vagotomisé et de surcroît diabétique, le délai imposé par la vagotomie n'est pas modifié, mais la durée du phénomène est encore abrégée (RUCKEBUSCH et BUENo, I977).

Chez nos porcs consommant du lactose, 1'hyperactivité post-prandiale n'est ni différée, ni abrégée, mais tout simplement absente. Aussi, il est exclu d'évaluer les hypothèses en présence : L'absence d'organisation post-prandiale procède-t-elle de la diminution générale des activités irrégulières? Résulte-t-elle d'une in fluence, vagale à point de départ éventuellement digestif, liée par exemple à l'extrême fluidité des conten us digestifs? Est-elle la conséquence d'une insuffisance d'insulinosécrétion post-prandiale? Quelques éléments peuvent cependant être rassemblés en faveur de cette troisième hypothèse. Bien que la digestion du lactose conduise à une absorption de glucose plus importante ( 56 à $77 \mathrm{p}$. Ioo des sucres réducteurs) que ne le laisse prévoir sa composition (RERAT et al., I977; RERAT, CorRING et LAPLACE, I978), la quantité de gluoces absorbé reste au total assez limitée. Ein effet, la digestion du lactose est lente, et le taux d'absorption très faible ( $5 \mathrm{p}$. Ioo en $8 \mathrm{~h}$ pour $800 \mathrm{~g}$ de lactose ingéré) est inversement proportionnel aux quantités de lactose consommées (RERAT, I975). L'étalement dans le temps de l'absorption de cette petite quantité de glucose ne paraît donc guère compatible avec une réponse insulinique de quelque ampleur.

Enfin, s'il est vrai que les glucides ne sont pas les seuls nutriments susceptibles d'entraîner l'hyperactivité post-prandiale (DE WEVER et al., I977), on peut souligner que celle-ci existe après ingestion d'aliment semi-synthétique à base d'amidon de maïs (LAPLACE, I978). La composition de cet aliment, pour les constituants autres que 1'amidon, est identique à celle de 1'aliment semi-synthétique ici utilisé, pour ses composants autres que le lactose. L'absence de toute hyperactivité postprandiale ne peut donc être attribuée qu'au lactose, quel que soit l'instrument de son effet, et non à quelqu'autre nutriment apporté par le régime.

\section{b) Activités régulières et irrégulières}

Depuis les travaux de Code et Schleger, (r973), Summers et Herm (r974), puis de Bueno, Fioramonti et Ruckebusch (I975) chez le Mouton et le Chien, et DARDILLAT (I975) chez le Veau, il est admis que les phases d'activité irrégulière et régulière ont un rôle à l'égard du déplacement des contenus digestifs. La propulsion des digesta survient au cours de la phase d'activité irrégulière et plus particulièrement à la fin de celle-ci, c'est-à-dire dans les instants qui précèdent en un site donné l'arrivée d'une activité régulière. Cette dernière ne transporte par ellemême aucun contenu. Elle pourrait constituer une barrière de pression limitant un éventuel reflux ou bien selon l'hypothèse de CODE et SCHLEGEL (I973) exercer une fonction de balayage. Cette description générale du rôle respectif des activités régulières et irrégulières à l'égard des mouvements de contenus digestifs, est également applicable au Porc (RERAT, CoRRING et LAPLACE, I978). Les relations entre les électromyogrammes intestinaux et les débits de digesta ont été récemment 
décrits de façon très complète dans le cas du Veau par DARdil, at (I977). Cet auteur souligne que l'intestin grêle est pratiquement vide pendant $80 \mathrm{p}$. Ioo du temps alors qu'il est presque en permanence le siège de phénomènes électriques.

Par rapport à ces informations, il est remarquable qu'après ingestion de lactose chez le Porc, des volumes de digesta extrêmement importants franchissent l'intestin grêle sur un mode relativement continu (et non par périodes de débits superposables aux activités irrégulières) et ce alors que les électromyogrammes sont particulièrement faibles. L'absence d'activités régulières sur les sites distaux ou leur raréfaction constitue un aspect de la réduction globale des activités rapides sur lequel nous reviendrons en relation avec les modifications de la migration des complexes.

Les activités irrégulières dont l'intensité est réduite de façon considérable et durable après ingestion de lactose sont associées dans le temps avec le déplacement rapide de volumes inhabituellement grands de contenus. Ce fait constitue une sorte de paradoxe. En effet chez le Porc consommant un régime standard, dont les volumes de contenus intestinaux sont très inférieurs, et chez lequel les débits de digesta surviennent par salves superposables aux activités irrégulières (fig. 5 et in RERAT, CORRING et LAPIACE, I978), il n'y a pas de corrélation entre 1'activité intestinale telle que quantifiée par intégration minute par minute à $2 \mathrm{~cm}$ en amont d'une fistule et le volume de contenus émis à la canule dans chaque unité de temps de I mn (LAPlace, données non publiées). Ceci confirme chez le Porc l'observation de DARDILLAT (I977) chez le Veau et traduit l'existence de nombreuses salves de potentiels d'action, saisies par l'intégration, qui ne correspondent pas à un débit. Après un repas d'épreuve au lactose, il semblerait à l'inverse que les débits de contenus soient démesurément importants relativement aux activités irrégulières enregistrées.

La modification des activités irrégulières après ingestion de lactose doit être également envisagée du point de vue de leur durée. Les résultats obtenus montrent que l'état de réplétion de l'intestin grêle est exceptionnel. Nous avons constaté antérieurement chez le Porc (LAPLACE, I978) que les phases d'activité irrégulière sont d'autant plus longues que la réplétion intestinale est importante. La décroissance de leur durée au long des $75 \mathrm{p}$. I oo proximaux de l'intestin grêle répond à la diminution de la quantité de contenus présents dans la lumière au fil du déroulement du transit chez le porc consommant un aliment standard. I a régularité de ce phénomène est modifiée au niveau iléal par le phénomène normal d'accumulation. On note dans le même sens que la réduction de la durée des activités irrégulières en un même site au cours de la période nocturne correspond, en cette période de repos alimentaire (lors de repas diurne) à la forte réduction des quantités résiduelles évacuées par l'estomac. Cette relation directe entre la durée des activités irrégulières et la réplétion intestinale est confirmée chez le Mouton par RuckEBusch et BuENo (I977) au travers d'expériences de dérivation vs. infusion de contenus digestifs. Selon ces auteurs cette relation reposerait sur des réflexes locaux, le pneumo-gastrique n'intervenant que dans l'ajustement des phénomènes. De même DARDILLAT (r977) note chez le Veau l'augmentation parallèle des débits intestinaux et de la durée des activités irrégulières. A l'opposé de ces informations, la réplétion de l'intestin grêle après consommation de lactose ne modifie pas la durée des activités irrégulières pour les régions proximales. Elle entraîne certes leur allongement apparent en région distale en l'absence des activités régulières et d'une organisation normale, ce qui interdit d'évaluer l'authenticité de la relation réplétion accrue-durée augmentée.

En définitive, il semblerait d'après les données recueillies dans ce travail 
que l'ingestion de lactose conduise à une augmentation considérable des volumes de contenus endoluminaux sans que la durée des activités irrégulières soit systématiquement allongée; ces volumes importants transitent rapidement en présence d'activités électriques exceptionnellement faibles et disparates. Ces observations viennent renforcer les questions qui se posent quant à l'exacte relation entre phénomènes électriques de la paroi intestinale et déplacement des contenus. Elles ajoutent d'autres interrogations relatives à la nature de la réplétion : les relations doivent-elles être recherchées en fonction du seul volume des contenus digestifs ou plutôt en fonction de leur volume, de leur teneur en matière sèche et de la nature de cette matière sèche.

\section{3. - Modifications de la migration des complexes}

Les modifications de la migration des complexes, évaluées au travers de la migration des activités régulières, consistent d'une part en une fréquence très importante de migrations limitées à une partie seulement de l'intestin grêle, d'autre part en l'accélération de la migration de ces activités. Les migrations partielles ne sont qu'exceptionnellement observées chez l'animal ingérant un régime standard (LAPLACE, I978), et principalement en période diurne, ce qui suggère une certaine influence de la réplétion. Cependant leur fréquence très supérieure de manifestation lors de consommation d'un régime riche en amidon de maïs, associée à une relative lenteur de la migration des complexes, a été attribuée (L,APLACE, I978) à un effet de la composition de l'aliment. Ceci a conduit à s'interroger sur un rôle éventuel de la réponse insulinique post-prandiale. Si 1'hypothèse reste à démon trer dans le cas de l'amidon de maïs, elle paraît inapplicable au cas du lactose.

En effet, dans ce dernier cas, les migrations partielles s'effectuent à une vitesse accélérée et non ralentie comme c'est le cas pour l'amidon de maïs. Les phénomènes observés pour ces 2 régimes ne sont donc pas entièrement comparables ce qui peut exclure une in fluence des fractions communes aux 2 aliments, autres que 1'amidon ou le lactose selon le cas. Indépendamment de cette différence de vitesse des migrations partielles, ce qui a été précédemment rappelé des processus d'absorption après ingestion de lactose, exclut une quelconque influence insulinique. Au demeurant, il paraîtrait curieux que l'efiet du lactose soit d'autant plus intense que la quantité ingérée est plus importante puisque la quantité absorbée de produits d'hydrolyse reste invariable quelle que soit la masse de lactose consommée par l'animal (RERAT, I975). Par conséquent, s'il reste plausible que la suspension de la migration des complexes myoélectriques soit la conséquence de la composition particulière du régime, l'instrument n'en est pas ici l'insuline, mais quelque autre facteur humoral ou nerveux, et les effets des aliments semi-synthétiques amidon de maïs ou lactose ne peuvent être superposés. Aux questions qui restent ainsi en suspens s'ajoute une interrogation quant au rôle possible de la réplétion très inhabituelle de l'intestin grêle.

Enfin il est malaisé d'évaluer les conséquences spécifiques de cette suppression de nombreuses activités régulières à l'égard du transit des contenus. L'observation des enregistrements montre que 1'absence d'activité régulière est associée à une absence de quiescence. La suppression des 2 phases d'activité au cours desquelles n'est déplacé aucun contenu, au profit des seules activités irrégulières ne peut, en dépit de la modicité des phénomènes électriques observés pendant le déroulement de ces dernières, que favoriser un transit accéléré. Un second point tient au fait que les activités régulières, normalement au nombre de 2 à 3 simulta- 
nément sur l'ensemble de l'intestin grêle du Porc (LAPLACE, I978) comme du Veau (DARDILIAT et MARRERo, I977) délimitent par leur présence et leur vitesse de migration l'ordre de grandeur de la vitesse de transit dans l'intestin grêle. L'absence d'activités régulières, et l'accélération de leur migration lorsqu'elles existent, ne peuvent donc que concourir à la continuité du débit dans l'intestin. A l'inverse, le ralentissement de la migration des activités régulières en période terminale de récupération, joint à l'éventuelle multiplication, à intervalles brefs, de ces activités lors de leur réapparition, peut être interprêté comme un phénomène compensateur après le passage d'un flot inhabituellement important de digesta.

\section{4. -- Ingestion de Lactose et diarrhée}

Les quelques observations concernant la motricité cæcale suggèrent, l'hyperactivité cacale réflexe liée au repas mise à part, deux étapes dans les modifications constatées chez les porcs ayant ingéré du lactose. Une période d'hypomotilité apparaît relativement précocement après la première ingestion de lactose. Il serait intéressant de savoir si ce phénomène est d'ordre réflexe, associé à la réduction des activités électriques de l'intestin grêle, ou s'il s'agit d'un effet local du lactose. On ne peut en effet exclure cette possibilité d'action directe qui implique un franchissement très rapide de l'intestin grêle. Les premières vagues de contenus liquides sont recueillies aux deux tiers de la longueur de l'intestin grêle moins d'une heure après le début du repas d'épreuve. La seconde étape dans les modifications observées consiste à l'inverse en une hypermotilité cæcale qui pourrait être attribuée, selon les données classiques rappelées précédemment, à l'accumulation de 1'acide lactique résultant de la fermentation du lactose.

Enfin, la diarrhée a été observée au bout d'un délai compris entre I 5 et $24 \mathrm{~h}$ et avec une intensité apparente très variable, chez tous les porcs utilisés. Il n'est cependant pas possible d'établir une relation claire entre la quantité de lactose ingéré et la précocité ou l'intensité de la diarrhée. Il nous a par ailleurs été donné d'observer depuis un animal qui n'a jamais présenté de diarrhée lors de 3 épreuves avec distribution de 2 repas apportant I ooo g de lactose chacun. L'état de vacuité digestive préalable au repas d'épreuve paraît également important. En effet après un repas-test unique à base de lactose distribué au terme d'un jêtne de $\mathrm{I} 6$ à $22 \mathrm{~h}$ (RERAT, I975), la diarrhée est soit beaucoup plus précoce (entre la $3^{\mathrm{e}}$ et la $8^{\mathrm{e}}$ heure après le repas) que chez nos porcs, soit absente chez certains animaux (RERAT, communication personnelle).

Par conséquent, il semblerait qu'il existe deux catégories de porcs dont la susceptibilité digestive à l'égard du lactose est très différente. Il n'est pas exclu que des sensibilités intermédiaires très diverses soient responsables de l'absence apparente de relation entre la quantité de lactose ingéré et la précocité et/ou 1'intensité de la diarrhée.

\section{Conclusion}

Ces observations préliminaires sur les effets du lactose à l'égard des complexes myoélectriques de l'intestin grêle du porc font surgir nombre de questions quant aux relations réelles entre phénomènes électriques et débits de contenus digestifs. On constate en particulier que le transit rapide de volumes importants de digesta très liquides est réalisé en présence d'activités irrégulières dont la durée 
n'est pas systématiquement augmentée, et dont l'intensité est exceptionnellement faible. La migration des activités régulières est très perturbée : ces activités migrent très rapidement mais ne parcourent que des segments limités d'intestin grêle; elles sont abolies au niveau distal durant des périodes atteignant $20 \mathrm{~h}$. Enfin la durée et l'intensité de la diarrhée consécutive ne sont pas clairement reliées à la quantité de lactose ingéré, probablement en raison de larges variations individuelles de la tolérance digestive au lactose.

Accepté pour publication en mai 1978.

\section{Remerciements}

Ce travail a bénéficié pour sa réalisation de l'aide de la D.G.R.S.T. (Division du fond de la recherche) dans le cadre de l'action sur programme "Interrelations aliments - tube digestif " (Décision 77-7-0463).

\section{Summary}

\section{Preliminary observations on some effects of lactose feeding in the pig: myoelectrical complexes in the small intestine, caecal motility and diarrhoea.}

The effects of ingesting a semi-synthetic diet containing $82 \mathrm{p}$. Ioo pure lactose on the organization of the electrical activities in the small bowel were studied in 5 Large white pigs weighing, on an average, $57 \mathrm{~kg}$ and fitted with permanent intraparietal supple electrodes. In another pig a reentrant cannula was placed in the distal part of the jejunum in order to check and evaluate the effect of this diet on the volumes of digesta passing through the gut.

The organization of spiking activities in the duodenum was normal. But, the aboral migration of the consecutive phases of the myoelectrical complexes was greatly disturbed. In the jejuno-ileal area regular spiking activities appeared and disappeared in a relatively anarchlc manner. An overall decrease in the regular spiking activities and even a total absence of activity in the ileum for periods of at least $5 \mathrm{~h}$ and maximum $2 \mathrm{rh}$, was observed. The higher the lactose intake level the longer lasted this effect. Parallel to that the intensity of the irregular spiking activities considerably decreased. However, in the jejunum we noticed a rapid transit of a 2 times larger volume of digesta than with a standard diet and a 3 to 4 times larger volume than with a maize starch diet.

These disturbances cannot be easily explained either by an insulin effect or by the only effect of an intestinal repletion like that occurring in other feeding conditions.

\section{Références bibliographiques}

Aumaitré A., Février C., Dewulf H., 1977. Influence du lactose alimentaire sur le développement de l'activité lactasique dans l'appareil digestif du Porc. C. R. Acad. Sci., 284 D, $2 \mathrm{I} 35^{-2} \mathrm{I} 38$.

BAILEY C. B., KITTS W. D., Woods A. J., I956. The development of the digestive enzyme system of the pig during its preweaning phase of growth. B. Intestinal lactase, sucrase and maltase. Canad. J. Agric. Sci., 36, 5 $1-58$.

Bueno L., Fioramonti J., RUCKebusch X., 1975. Rate of flow of digesta and electrical activity of the small intestine in dogs and sheep. J. Physiol., Londres, 249, 69-85.

BUENO L., WEEKES T. E. C., RUCKEBUSCH Y., I977. Effects of diet on the motility of the small intestine and plasma insulin levels in sheep. Ann. Rech. V'et., 8, 95-I04. 
CHristophe, N. L., BAyless T. M., I97 I. Role of the small bowel and colon in lactose-induced diarrhea. Gastroenterology, 60, 845-862.

CODE C. F., MARLE'T' J. A., I975. The interdigestive myoelectric complex of the stomach and small bowel of dogs. J. Physiol., Londres, 246, 289-309.

CODE C. F., SCHLEGEL J. F., I973. The gastrointestinal interdigestive house keeper : motor correlates of the interdigestive myoelectric complex of the dog. In 4 th Int. Symp. on Gastrointestinal Motility, pp. 63-64, Mitchell Press, Vancouver, Canada.

Dahlquist A., I96r. The location of carbohydrases in the digestive tract of the Pig. Biochem. $J ., 78,282-288$.

DARDILIAT C., I975. Gastrointestinal motility in calf neonatal disease in RuTTER J. M., Perinatal ill health in calves, pp. xr I-122. Simpsons Press, Newbury, Berkshire (U. K.).

Dardirlat C., I977. Analyse électromyographique et débitmétrique du transit alimentaire chez le Veau nouveau-né. J. Physiol., Paris, 73, 925-944.

Dardilitat C., Marréro E., i977. Eitude de l'électromyogramme global chronique de la paroi intestinale du veau préruminant : migration des phases d'activité régulière et relation avec le transit. Ann. Biol. anim. Bioch. Biophys., 17, 523-530.

Debongnie J. C., Newcomar A. D., Phirlips S. F., i977. Small bowel function in lactase deficiency. Gastroenterology, 72, $\mathrm{A} 23-1046$.

De Wever I., Eeckhout C., Hellemans J., Vantrappfin G., 1977. The effect of feeding on the interdigestive myoelectric (migrating) complex (IDMEC) in dogs. in 6th Int. Symp. on Gastrointestinal Molitity, Edinburgh, Scotland, I2-I6 september, p. 6 .

EKSTROM K. E., BENEVENGA N. J., GRUMmER R. H., r975. Effects of diets containing dried whey on the lactase activity of the small intestinal mucosa and the contents of the small intestine and cecum of the Pig. J. Nutr., 105, 85 I-860.

Ekstrom K. E., Grummer R. H., BenevenGA N. J., I976. Effects of a diet containing 40 p. Ioo dried whey on the performance and lactase activities in the small intestine and cecum of hampshire and chester with pigs. J. anim. Sci., 42, I06-II3.

Fayart G., Daburon F., LAPLaCe J. P., i978. Étude d'un électromyographe numérique multivoies. C.E.A., Notice technique C.E.A.-N.T.-55. Janvier 1978.

Hartman P. A., Hays V. W., Baker R. O., Neagle L. H., Catron D. V., i96r. Digestive enzyme development in the young pig. J. anim. Sci., 20, II4-I23.

HELLER M. D., KERN F., I968. Absorption of lactic acid from an isolated intestinal segment in the intact rat. Proc. Soc. exp. Biol. Med., 127, I I03-rio6.

KFRN F., STRUTHERS J. E., I966. Intestinal lactase deficiency and lactose intolerance in adults. J. am. med. assoc., 195, 927-93o.

Koldovsky O., r966. The postnatal development of enzyme activities of the sniall intestine. in : Development of metabolism as related to Nutrition, Karger ed., Basel, I77 pp.

Koldovsky O., ChyTIL, F., I 965. Post natal development of $\beta$-galactosidase activity in the small intestine of the Rat. Biochem. J., 94, 266-270.

LAPLACE J. P., r978. Organisation de la motricité de l'intestin grêle chez le Porc et influence de l'alimentation. Ann. Zootech., 27, 377-408.

LAPlace J. P., Tomassone R., I97o. Évacuation gastro-duodénale chez le Porc. Fistulation chronique par voie transthoracique extrapleurale : recherche d'une technique d'analyse mathématique de 1'évacuation. Ann. Zootech., 19, 303-332.

LATOUR A., I973. Un dispositif simple d'analyse quantitative de l'électromyogramme intestinal chronique. Ann, Rech. Vet., 4, 347-353.

LAUNIALA K., r968. The mechanism of diarrhea in congenital dissacharise mal-absorption. Acta paediatr. scand., 57, 425-432.

Manners M. J., Stevens J. A., I972. Changes from birth to maturity in the pattern of distribution of lactase and sucrase activity in the mucosa of the small intestine of pigs. $B r . J$. Nutr., 28, Ir3-I27.

PARsons D. S., PATERson C. R., I965. Fluid and solute transport accross rat colonic mucosa. Quarl. J. exp. Physiol., 50, 220-231.

RERAT A., I975. Mesure quantitative in vivo de l'absorption chez le Porc. Application aux sucres et aux acides aminés. In : Réanimation entérale à faible débit continu, Colloque INSERM, 20 nov. I975, vol. 53, pp. 47-62.

Rerat A., Aumaitre A., Vaissade P., Vaugelade P., I977. Étude expérimentale qualitative de l'absorption des glucides après ingestion d'un repas à base de lactose chez le Porc. Ann. Biol. anim. Bioch. Biophys., 17, 589-596. 
Rerat A., Corring T., Laplace J. P., I978. Quelques aspects des recherches en plyysiologie digestive chez le Porc. Applications possibles. Journées Rech. porcine cu Irance, I.N.R.A.I.T.P. Ed., Paris, pp. 95-1 18.

Rousseau B., Sladen G. F., I97I. Fffect of luminal pH on tlie absorption of water, Na + and $\mathrm{Cl}$-.. by rat intestine in vivo. Biochem. Biophys. Acta, 233, 591-593.

RuCkebusch Y., BUeno L., I977. The migrating myoelectric complex of the small intestine. An intrinsic activity mediated by the vagus. Gastroenterology 73, I309-I3I4.

Ruckebusch Michèle, Fronamontr J., I975. Insulino-sécrétion et motricité intestinale. C.R. Soc. Biol., 169, 435-439.

Summers R. W., HeI,M J., I974. The effect of feeding and the myoelectric complex on transit in the canine small intestine, Gastroenterology, 66, 786 (Abstr.).

WALKER D. M., I959. The development of the digestive system of the young animal. 2) Carbohydrase enzyme development in the young pig. J. Agric. Sci., 52, 357-37o.

Weijers H. A., VAN de Kamer J. H., Dicke W. K., IJsseling J., r96i. Diarrhea caused by deficiency of sugar splitting enzymes. Acta paediatr. (Upps.), 50, 55-7 I.

YEH K. Y., MOOG F., I974. Intestinal lactase activity in the suckling rat: influence of hypophysectomy and thyroïdectomy. Science, 183, 77-79. 